\title{
Avandia: Cardiovascular Safety Concern Remains
}

\section{Chun Jiang*}

Department of Biology, Georgia State University, Atlanta, Georgia 30302-4010, USA

\section{Introduction}

Avandia or rosiglitazone is widely used for the treatment of Type2 diabetes (T2D), marketed by the GlaxoSmithKline Pharmaceuticals. It has several beneficial effects on the pathogenesis of diabetes as well as its complications: 1) By improving insulin resistance Avandia has been successfully used to achieve glycemic control in a manner that is at least as effective as sulfonylureas and metformin; 2) via PPAR $\gamma$ Avandia regulates adipocyte proliferation and lipid storage, improving lipid profile; 3) Avandia interferes with the foam cell formation and inflammatory response, reduces lipid deposition in the vessel wall, and thus attenuates the development of atherosclerosis [1-3].

In 2007, a large meta-analysis suggests a $43 \%$ increase in risk for myocardial infarction and a possible increase in risk for cardiovascular death with Avandia treatment [4]. This was followed by a number of clinical trials. Because results from these studies were rather inconsistent, the Food and Drug Administration (FDA) decided not to withdraw the drug from the market in 2007 and 2010. Meanwhile a safety alert was issued, warning a possibly increased risk of ischemic cardiovascular events in patients using Avandia. Subsequently, the FDA required a reevaluation of the Rosiglitazone Evaluated for Cardiac Outcomes and Regulation of Glycaemia in Diabetes (RECORD) trial. With new RECORD data, the FDA has lifted use restrictions of Avandia in November, 2013 [5]. A recent article in New England Journal Medicine concluded: "From a cardiovascular perspective, rosiglitazone, saxagliptin, and alogliptin appear to be relatively safe [1]."

However, the RECORD trials were designed to evaluate whether in the treatment of T2D Avandia was interior to metformin/sulfonylurea on cardiovascular outcomes [6]. Although such interiority was not found, the RECORD results should not be interpreted as Avandia does not elevate cardiovascular risks. The RECORD results only suggest that the cardiovascular risks in Avandia users are about the same as in metformin/sulfonylurea users. Indeed, a recent metaanalysis of randomized clinical trials indicates that the use of sulfonylureas in T2D is associated with increased mortality and a higher risk of stroke [7].

Experimental evidence from bench research suggests that Avandia has adverse effects on the vascular wall, compromising vasodilation responses to circulating hormones and neurotransmitters as well as metabolites. Such adverse effects are independent of PPAR $\gamma$ and involve the vascular KATP channel that is inhibited by Avandia and sulfonylureas [8-10]. Since the RECORD trials only compared cardiovascular risks between Avandia and sulfonylureas/metformin, the RECORD results should be interpreted with great precautions.

The information of the novel vascular target seems to explain several adverse cardiovascular effects of Avandia and may be helpful for physicians and diabetic patients to use the drug more properly by taking the advantage of its metabolic benefits and avoiding potential cardiovascular risks [11]. The finding should not be considered as evidence to indicate that Avandia is a bad drug, as no drugs are completely free of side-effects including widely used Aspirin, Prozac and Vitamins.

\section{References}

1. Hiatt WR, Kaul S, Smith RJ (2013) The cardiovascular safety of diabetes drugs-insights from the rosiglitazone experience. N Engl J Med 369: 12851287.

2. Irons BK, Greene RS, Mazzolini TA, Edwards KL, Sleeper RB (2006) Implications of rosiglitazone and pioglitazone on cardiovascular risk in patients with type 2 diabetes mellitus. Pharmacotherapy 26: 168-181.

3. Barnett $\mathrm{AH}$ (2009) Redefining the role of thiazolidinediones in the management of type 2 diabetes. Vasc Health Risk Manag 5: 141-151.

4. Nissen SE, Wolski K (2007) Effect of rosiglitazone on the risk of myocardia infarction and death from cardiovascular causes. N Engl J Med 356: 24572471.

5. Mitka M (2013) FDA eases restrictions on the glucose-lowering drug rosiglitazone. JAMA $310: 2604$

6. Home PD, Pocock SJ, Beck-Nielsen H, Gomis R, Hanefeld M et al. (2005) Rosiglitazone Evaluated for Cardiac Outcomes and Regulation of Glycaemia in Diabetes (RECORD): study design and protocol. Diabetologia 48: 1726-1735.

7. Monami M, Genovese S, Mannucci E (2013) Cardiovascular safety of sulfonylureas: a metaanalysis of randomized clinical trials. Diabetes Obes Metab 15 : 938-953.

8. Yu L, Jin X, Yang Y, Cui N, Jiang C (2011) Rosiglitazone inhibits vascular KATP channels and coronary vasodilation produced by isoprenaline. $\mathrm{Br} \mathrm{J}$ Pharmacol 164: 2064-2072.

9. Lei Yu, Xin Jin, Ningren Cui, Yang Wu, Zhenda Shi, Daling Zhu et al. (2012) Rosiglitazone selectivelyinhibits $\mathrm{K}(\mathrm{ATP})$ channels by acting on the $\mathrm{K}(\mathrm{IR}) 6$ subunit. Br J Pharmacol 167: 26-36.

10. Dart C (2012) Selective block of K(ATP) channels: why the anti-diabetic sulphonylureas and rosiglitazone have more in common than we thought. $\mathrm{Br}$ Pharmacol 167: 23-25

11. Jiang C, Yu L, Jin X, Wang YJ (2014) Rosiglitazone acts on the vascula KATP channel and compromises coronary circulation. In: Handbook of Type 2 Diabetes in the Middle Aged and Elderly: Pharmacology, Clinical Uses and Adverse Effects. Advances in Medicine and Biology. Vol 79 Ed: Leon V. Berhardt, Nova Science Publishers. pp. 1-8

Received September 29, 2015; Accepted November 22, 2015; Published November 25,2015

Citation: Jiang C (2015) Avandia: Cardiovascular Safety Concern Remains. J Mo Biomark Diagn 6: 258. doi:10.4172/2155-9929.1000258

Copyright: (C) 2015 Jiang C. This is an open-access article distributed under the terms of the Creative Commons Attribution License, which permits unrestricted use, distribution, and reproduction in any medium, provided the original author and source are credited. 